\title{
Three-drug combination of lacosamide, phenobarbital and valproate exerts additive interaction in the tonic-clonic seizure model in mice
}

\author{
Maria Kondrat-Wróbel ${ }^{1, B-C, F} \oplus$, Paweł Marzęda ${ }^{1, B-C, F \oplus}$, Hubert Bojar ${ }^{2, B, D, F} \oplus$, \\ Paula Wróblewska-Łuczka ${ }^{1, C, E-F \oplus}$, Justyna Kozińska ${ }^{3, B-C, F \oplus}{ }^{\oplus}$ Marek Jankiewicz ${ }^{4, B-D, F}{ }^{\oplus}$, \\ Mateusz Kominek ${ }^{5, B, D, F}$, Jarogniew J. Łuszczki ${ }^{1,6, A, D-F} \oplus$ \\ ${ }^{1}$ Department of Pathophysiology, Medical University, Lublin, Poland \\ ${ }^{2}$ Department of Toxicology and Food Safety, Institute of Rural Health, Lublin, Poland \\ ${ }^{3}$ Department and Clinic of Hematolooncology and Bone Marrow Transplantation, Medical University, Lublin, Poland \\ ${ }^{4}$ Department and Clinic of Cardiology, Medical University, Lublin, Poland \\ ${ }^{5}$ Department of Orthopedics and Traumatology, Medical University, Lublin, Poland \\ ${ }^{6}$ Isobolographic Analysis Laboratory, Institute of Rural Health, Lublin, Poland \\ A - Research concept and design, B - Collection and/or assembly of data, C - Data analysis and interpretation, \\ $D$ - Writing the article, E-Critical revision of the article, F- Final approval of article
}

Kondrat-Wróbel M, Marzęda P, Bojar H, Wróblewska-Łuczka P, Kozińska J, Jankiewicz M, Kominek M, J. Łuszczki J. Three-drug combination of lacosamide, phenobarbital and valproate exerts additive interaction in the tonic-clonic seizure model in mice. J Pre Clin Clin Res. 2020; 14(3): 102-106. doi: $10.26444 / j p c c r / 127377$

\section{Abstract}

Introduction. Triple-therapy with antiepileptic drugs (AEDs) is usually prescribed for epilepsy patients, whose seizures are not fully controlled with standard medications. Although 25 various AEDs are currently licensed for treating epilepsy, no algorithms allowing for the proper combination of AEDs are available.

Objective. The aim of the study is to isobolographically assess the type of interaction among three AEDs (lacosamide [LCM], phenobarbital [PB] and valproate [VPA]), in the model of tonic-clonic seizures in mice.

Materials and Method. The electrically-evoked $(25 \mathrm{~mA}, 500 \mathrm{~V}, 50 \mathrm{~Hz}, 0.2 \mathrm{~s}$ of stimulus duration) tonic-clonic seizures in male albino Swiss mice allowed determination of the anticonvulsant action of the three-drug mixture of LCM, PB and VPA combined in a dose ratio of 1:1:1 by means of type I isobolographic analysis of interaction.

Results. The experimentally-determined ED50 exp value for the three-drug mixture was $112.04 \mathrm{mg} / \mathrm{kg}$ and did not differ from the theoretically calculated ED50 add value, which was $112.36 \mathrm{mg} / \mathrm{kg}$. Lack of statistical significance confirmed that the mixture of LCM, PB and VPA in a dose-ratio of 1:1:1 exerted additive interaction in the mouse tonic-clonic seizure model. Conclusions. Although the three-drug combination of LCM, PB and VPA produced additive interaction in the mouse tonic-clonic seizure model, the three-drug combination could be recommended for epilepsy patients whose seizures are refractory to the standard medication.

\section{Key words}

valproate, drug interactions, phenobarbital, isobolographic analysis, lacosamide, Tonic-clonic seizures

\section{INTRODUCTION}

Relatively recently, two novel antiepileptic drugs (AEDs, cenobamate and perampanel, have been licensed and approved as add-on drugs to clinical practice by FDA in USA and EMA in the EU $[1,2]$. At present, physicians can prescribe for epileptic patients approx. 25 various AEDs; however, these drugs can offer seizure freedom in only approx. $70 \%$ of epileptic patients, while the rest of the patients remain refractory to standard treatment $[3,4]$. To help the patients in eliminating seizure attacks, physicians have to take into consideration the application of some novel AEDs and/or various combinations of AEDs [5]. At present, only a few combinations of AEDs are favourable in

Address for correspondence: Jarogniew J.Łuszczki, Department of Pathophysiology Medical University, 20-090 Lublin, Poland

E-mail: jarogniew.luszczki@umlub.pl

Received:30.07.2020; accepted:08.09.2020; first published: 14.09.2020 epilepsy patients [5]. The choice of AEDs in the respective combinations is still a challenging issue for doctors, who must have comprehensive knowledge on AEDs and clinical experience in treating patients with AEDs [5]. Taking into account an ample number of available AEDs (25 or more), it is difficult to properly choose the AEDs for combinations. In such cases, preclinical studies based on isobolographic analysis of interaction can readily help clinicians in their choice and selection of AEDs [6-8].

Accumulating experimental evidence indicates that some triple combinations of AEDs may produce not only synergistic, additive, but also antagonistic interaction [9-11]. There is no doubt that only AEDs in combinations exerting synergistic and additive interactions, can be recommended for patients with refractory epilepsy [5]. On the other hand, it is not possible to theoretically predict the exact types of interactions for various three-drug combinations of AEDs in animals. 


\section{OBJECTIVE}

The aim of this study was to determine the exact type of interaction among three AEDs: lacosamide (LCM), phenobarbital (PB), and valproate (VPA) by means of isobolographic analysis of interaction, as recommended elsewhere $[9,10,12]$. Due to various molecular mechanisms of action of the studied AEDs, the three-drug combination of LCM+PB+VPA should theoretically exert supra-additivity (synergy) in the mouse tonic-clonic seizure model. Each beneficial combination of AEDs, verified in preclinical studies, could become a new method of treatment, providing the epileptic patients with a state of seizure freedom.

\section{MATERIALS AND METHOD}

Animals. After a week of acclimatization to laboratory conditions, 32 adult male albino Swiss mice (weighing 22$26 \mathrm{~g}$ ) were subjected to experimental protocol, approved by the Second Local Ethics Committee at the University of Life Sciences in Lublin, Poland. Experiments were performed in strict accordance with the ARRIVE guidelines [13].

Drugs. LCM (Vimpat, UCB Pharma SA, Brussels, Belgium) and PB (Sigma-Aldrich, Poznań, Poland) were suspended in a $1 \%$ aqueous solution of Tween 80 (Sigma-Aldrich, Poznań, Poland). Only VPA (sodium salt - Sigma-Aldrich, Poznań, Poland) was dissolved in sterile distilled water. The drugs were injected intraperitoneally: LCM at $15 \mathrm{~min}, \mathrm{~PB}$ - at 60 min and VPA - at $30 \mathrm{~min}$, before the electrically-evoked tonic-clonic seizures, as described elsewhere $[9,14,15]$.

Tonic-clonic seizure model in mice. Tonic-clonic seizures in 32 mice were evoked electrically using alternating current $(25 \mathrm{~mA}, 500 \mathrm{~V}, 50 \mathrm{~Hz}, 0.2 \mathrm{~s}$ stimulus duration) delivered via auricular electrodes. The animals were randomly divided into 4 groups (8 mice in each group), which were given injections of LCM, PB and VPA in a constant combination of dose ratio of 1:1:1. After the respective pretreatment times, the animals were subjected to electroconvulsions. The number of the animals protected from tonic-clonic seizures in each experimental group, along with the increasing doses of the AEDs in combination, were linearly related according to the log-probit method [16]. The protective effects of the mixture of LCM, PB and VPA (when administered in the three-drug mixture in a dose ratio of 1:1:1) from tonic-clonic seizures were expressed as the $\mathrm{ED}_{50 \mathrm{mix}}$ (in $\mathrm{mg} / \mathrm{kg}$ ), as presented elsewhere $[6,17,18]$.

Isobolographic analysis and statistics. Isobolographic analysis is the method of choice when classifying pharmacodynamic interaction among the tested drugs. This method allows comparison of the experimentally derived effects for the drugs in mixture with the theoretically calculated pure additive effects offered by these drugs [19]. Statistical comparison of experimentally-derived values with purely additive values allowed differentiating the interaction from additivity. If the effects are higher than the additive effect, then synergy (supra-additivity) is reported [19]. If the observed effects are lower than the additive effect, antagonism (sub-additivity) is documented [19]. In the current study, isobolographic analysis was based on calculation of the
$\mathrm{ED}_{50}$ values, i.e., median experimental dose of three-drug mixture that protected $50 \%$ of the animals tested from tonicclonic seizures. The median additive dose of the drugs in the mixture that protected $50 \%$ of the animals from seizures, was calculated from the equation of additivity for three-drug mixture [20], as follows:

$$
\begin{gathered}
\mathrm{ED}_{50 \text { add }}=1 / 3 \mathrm{ED}_{50} \text { of } \mathrm{LCM}+1 / 3 \mathrm{ED}_{50} \text { of } \mathrm{PB}+1 / 3 \mathrm{ED}_{50} \\
\text { of VPA }
\end{gathered}
$$

In this case, the drug dose ratio for the three-drug mixture was constant and amounted to 1:1:1. With isobolographic analysis, the experimentally-derived $\mathrm{ED}_{50 \exp }$ value (in $\mathrm{mg} / \mathrm{kg}$ ) for the three-drug mixture of LCM, PB and VPA (in a dose ratio of $1: 1: 1)$ was statistically compared to the theoretically calculated $\mathrm{ED}_{50 \text { add }}$ value (in $\mathrm{mg} / \mathrm{kg}$ ), as recommended elsewhere $[8,19]$. The unpaired Student's $t$-test was used to perform statistical comparison between $\mathrm{ED}_{50 \exp }$ and $\mathrm{ED}_{50}$ values for the three-drug mixture of LCM, PB and VPA. Statistical significance was set up at $P<0.05$.

\section{RESULTS}

Isobolographic analysis of protective effects of the threedrug mixture from tonic-clonic seizures in animals. To calculate the $\mathrm{ED}_{50 \text { add }}$ value for the three-drug mixture of LCM, PB and VPA, the $\mathrm{ED}_{50}$ values of the AEDs was used when administered alone that amounted to $7.27 \pm 0.77 \mathrm{mg} / \mathrm{kg}$ for LCM; $31.21 \pm 2.04 \mathrm{mg} / \mathrm{kg}$ for PB and $298.63 \pm 15.15 \mathrm{mg} / \mathrm{kg}$ for VPA (Figure 1).

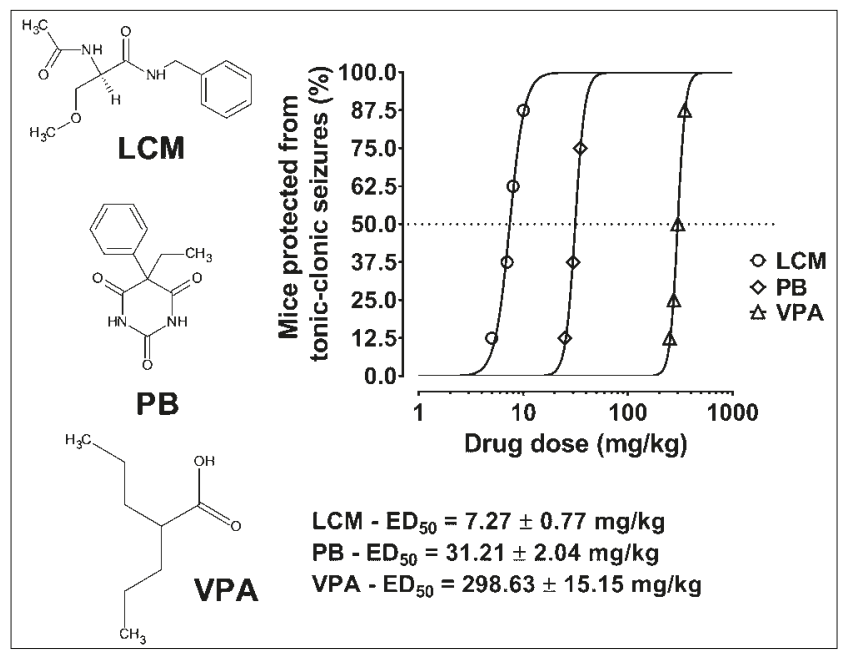

Figure 1. Structural formulas of lacosamide (LCM), phenobarbital (PB), valproate (VPA) and their protective effects from tonic-clonic seizures in mice

In such a situation, the theoretically calculated $\mathrm{ED}_{50 \text { add }}$ value was $112.36 \pm 5.10 \mathrm{mg} / \mathrm{kg}$. The three-drug combination of LCM, PB and VPA, in a dose-dependent manner, protected the animals from electrically-evoked tonic-clonic seizures; the experimentally-derived $\mathrm{ED}_{50 \exp }$ value was $112.04 \pm$ $9.43 \mathrm{mg} / \mathrm{kg}$ (Tab. 1).

Statistical comparison of both, $\mathrm{ED}_{50 \text { add }}$ and $\mathrm{ED}_{50 \exp }$ values revealed no significance (Fig. 2A-C). Isobolographic analysis of interaction revealed that the three-drug mixture of LCM, $\mathrm{PB}$ and VPA exerted additive interaction in the tonic-clonic seizure model in mice (Fig. 2A-C). 
Table 1. Protective effects of three-drug mixture in the mouse tonicclonic seizure model

\begin{tabular}{lcccc}
\hline Animals & \multicolumn{3}{c}{ Drug } & Anticonvulsant effect (\%) \\
\cline { 2 - 4 } & LCM & PB & VPA & \\
\hline Group I (n=8) & 1.83 & 7.85 & 75.10 & 12.5 \\
Group II ( $n=8)$ & 2.26 & 9.70 & 92.84 & 37.5 \\
Group III ( $\mathrm{n}=8)$ & 2.83 & 12.13 & 116.05 & 75 \\
Group IV (n=8) & 3.48 & 14.96 & 143.12 & 100 \\
\hline Fixed-ratio 1:1:1 & 2.42 & 10.40 & 99.53 & $\mathrm{ED}_{50 \text { add }}=112.36 \pm 5.10$ \\
Fixed-ratio 1:1:1 & 2.42 & 10.37 & 99.25 & $\mathrm{ED}_{50 \text { mix }}=112.04 \pm 9.43$ \\
\hline
\end{tabular}

Doses of particular AEDs (LCM, PB and VPA) are expressed in $\mathrm{mg} / \mathrm{kg}$.

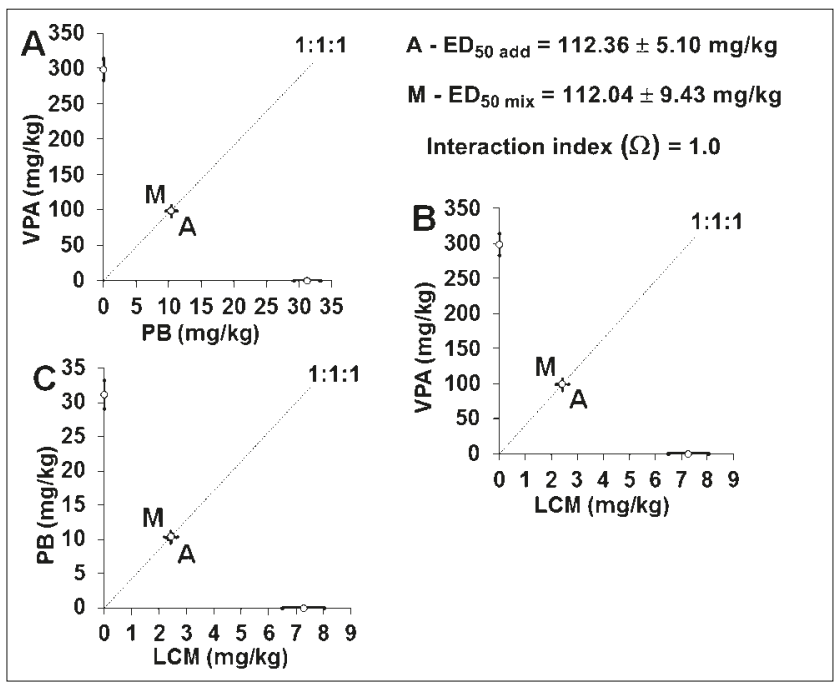

Figure $\mathbf{2 A - 2 C}$. Additive interaction for the combination of lacosamide (LCM) phenobarbital (PB) and valproate (VPA) in the tonic-clonic seizure model in mice. Values of ED50 ( \pm S.E.M.) for LCM, PB and VPA are graphically plotted on X and $Y$ axes, respectively. The points $A$ and $M$ represent the ED50 add and ED50 exp values, respectively. Since both points ( $A$ and $M$ ) overlap each other and are placed graphically in the same position, the interaction for three-drug combination of LCM, PB and VPA was purely additive in the mouse tonic-clonic seizure model

\section{DISCUSSION}

Results obtained in this study indicate that the combination of the three AEDs produced purely additive interaction in the mouse MES model. This combination of LCM+PB+VPA is worthy of being recommended for epilepsy patients whose seizures are refractory to the standard treatment by means of AEDs. No doubt exists that when three consecutive monotherapies with different current frontline AEDs failed, clinicians prescribe their patients polytherapy with two or more AEDs [21, 22]. The first presumption when choosing AEDs to combine them together is molecular mechanisms of action of the tested drugs. The main rule is to combine AEDs with diverse mechanisms of action so that the threedrug combination has several potential target sites; thus, the drugs could really have the potential to suppress seizures [22]. Multi-targeted antiseizure activity exerted by the triple AED combination minimizes the risk of ineffective treatment in epileptic patients. The second presumption when selecting the AEDs for the combination is their low (or no) potential to evoke adverse effects, especially, if they are combined together $[23,24]$. This can be achieved by reduction of drug doses used to suppress seizures. In the current study, both presumptions were fulfilled. The third presumption taken into account by physicians when choosing the AEDs for a combination is the favourable pharmacokinetic profiles exerted by the AEDs used in combination $[25,26]$. In such a case, the reduction of drug doses should also minimize or even eliminate pharmacokinetic drug-drug interactions among LCM, PB and VPA.

From a pharmacological viewpoint, LCM potentiates slow inactivation of voltage-gated sodium channels while simultaneously having no impact on the fast inactivation of these channels; thus, LCM inhibits repetitive neuronal firing and stabilizes hyperexcitable neuronal membranes that finally suppresses seizure generation in the pathologicallychanged brain tissue [27]. $\mathrm{PB}$ potentiates neuronal inhibition through the GABA $\mathrm{A}_{\mathrm{A}}$ receptors [28]. Regarding VPA, the drug blocks T-type calcium channels, increases GABA content in the brain, reduces NMDA-mediated excitatory neurotransmission and blocks high voltage activated sodium channels in neurons [29]. All the above-mentioned mechanisms can be involved in suppression of various forms of epilepsy. Besides, it is not clear whether one single mechanism of VPA can sufficiently diminish seizure activity in the epileptic brain, or several various VPA mechanisms must be simultaneously activated to terminate seizure attacks in the epileptic brain. Theoretically, bearing in mind all the molecular mechanisms of action produced by LCM, PB and VPA, there is no doubt that the combination comprising these three AEDs should exert favorable interaction in experimental animals.

The current study proves that the combination of $\mathrm{LCM}+\mathrm{PB}+\mathrm{VPA}$ is beneficial, although its pharmacodynamic interaction was additive in the mouse tonic-clonic seizure model. This combination is also a good example demonstrating that clinicians can combine drugs belonging to the first (PB and VPA) and third (LCM) generations of AEDs. Considering PB and its anticonvulsant profile in both humans and animals, the authors are aware of the fact that application of PB is clinically restricted to situations related with termination of neonatal and childhood seizures [28]. The drug is seldom prescribed to adult patients, except for drugresistant convulsive and non-convulsive status epilepticus [28], but in the presented study, $\mathrm{PB}$ was combined together with LCM and VPA, due to its unique molecular mechanisms action linked to $\mathrm{GABA}_{\mathrm{A}}$-receptor-mediated inhibition of neurotransmission in the brain.

Preclinical verification of interaction for the combination of LCM with PB and VPA confirmed the existence of pure additivity in terms of suppression of tonic-clonic seizures in animals, and the results are quite similar to those already published in the same seizure model for the three-drug combinations containing LCM (i.e., LCM+CBZ+LTG, $\mathrm{LCM}+\mathrm{CBZ}+\mathrm{PB}, \mathrm{LCM}+\mathrm{LTG}+\mathrm{PB}$ (Tab. 2) [30-32]. On the other hand, some three-drug combinations comprising LCM occurred antagonistic (i.e., LCM+CBZ+VPA and $\mathrm{LCM}+\mathrm{LTG}+\mathrm{VPA})$ in the mouse tonic-clonic seizure model (Tab. 2) $[9,10]$. At present, it is not clear why some combinations of three AEDs produce additivity while the others exert antagonistic interaction.

Of note, the supra-additive (synergistic) interactions among the three AEDs can be readily recommended for testing in clinical conditions. Also, the additive interactions among AEDs can be worthy of consideration when testing them in clinical settings. In the case of sub-additive (antagonistic) 
Table 2. Interactions for combinations of three AEDs in the model of tonic-clonic seizures in mice

\begin{tabular}{lcc}
\hline Combination of three drugs & Type of interaction & References \\
\hline CBZ + PB + TPM & supra-additivity & {$[33]$} \\
\hline OXC + PGB + TPM & supra-additivity & {$[11]$} \\
\hline PB + PHT + PGB & supra-additivity & {$[34]$} \\
\hline$L C M+C B Z+$ LTG & additivity & {$[31]$} \\
\hline$L C M+C B Z+P B$ & additivity & {$[30]$} \\
\hline$L C M+L T G+P B$ & additivity & {$[32]$} \\
\hline$C B Z+P B+V P A$ & additivity & {$[35]$} \\
\hline$L C M+L T G+V P A$ & sub-additivity & {$[9]$} \\
\hline$L C M+C B Z+V P A$ & sub-additivity & {$[10]$}
\end{tabular}

CBZ - carbamazepine; LCM - lacosamide; LTG - lamotrigine; OXC - oxcarbazepine; PB - phenobarbital; PGB - pregabalin; PHT - phenytoin; TPM - topiramate; VPA - valproate.

interactions among three AEDs, a special caution is advised for clinicians when combining these AEDs during the treatment of refractory patients in order not to expose them to ineffective treatment regimens [23].

It should be highlighted that in the presented study only isobolographic analysis of the interaction for the mixture of three AEDs injected singly in an acute model of experimental seizures was performed. No chronic administration of drugs in mixture was conducted in this study. Since the drugs were injected singly, there were no pharmacokinetic interactions associated with induction and/or inhibition of the drugs' metabolism pathways. It is noteworthy that both PB and VPA when administered chronically, mutually affect some liver CYP isoenzymes, contributing to substantial changes in drugs concentrations [29]. Besides, chronic administration of $\mathrm{PB}$ is associated with the development of tolerance to the drug dose in patients, which requires a substantial adjustment of drug doses to reach the same anticonvulsant effects [28].

The main limitation in this study was lack of determination of acute adverse effects produced by the three-drug mixture. However, results from previously published studies revealed that the doses of AEDs used in three-drug mixtures were generally too low to exert any acute adverse effects in experimental animals [10,30-32]. Previously, it was found that none of the studied AED combinations produced impairment in motor coordination in rodents or disturbed long-term memory in experimental animals. Additionally, skeletal muscular strength was also unchanged in experimental animals receiving three-drug combinations in doses reflecting the $\mathrm{ED}_{50 \mathrm{exp}}$ values from the mouse tonic-clonic seizure model [10,30-32]. Thus, the three-drug combination of $\mathrm{LCM}+\mathrm{PB}+\mathrm{VPA}$ seems to be safe enough, and therefore can be recommended to clinical practice for epileptic patients.

Another fact needs discussion and explanation while transferring the results from this study to clinical conditions. The isobolographic protocol for evaluating AED interactions refers to the activity of one AED because the equation of additivity is based on one fully active drug [20]. In contrast, in clinical conditions, epilepsy patients receive two or three AEDs in a full dose range each, providing them with duotherapies or triple-therapies [5, 36, 37].

Of note, the $\mathrm{ED}_{50}$ values for LCM, PB and VPA when used alone were determined in previous studies $[10,30,35]$ that allowed for calculating the $\mathrm{ED}_{50 \text { add }}$ value for the three-drug mixture. This was the reason that in the current study only
32 animals were used in the mouse tonic-clonic seizure model for determining the $\mathrm{ED}_{50 \text { exp }}$ value. Such a drastic reduction of tested animals was in strict accordance with the 3Rs rule (Reduction, Refinement and Replacement) of laboratory animals during the in vivo study [13]. The total number of animals used was reduced to a minimum in order to determine the $\mathrm{ED}_{50 \text { exp }}$ value for the combination of $\mathrm{LCM}+\mathrm{PB}+\mathrm{VPA}$ in a constant dose-ratio of 1:1:1.

It should be stressed that the main limitations in this study were both the route of drug administration and single application of the drugs. In clinical conditions, the AEDs are usually taken by the patients orally and chronically for a long period of time $[36,37]$. In this study, the AEDs were injected i.p. and only as single injections of LCM, PB and VPA.

\section{CONCLUSIONS}

1. LCM combined with PB and VPA produced additivity in the mouse model of tonic-clonic seizures.

2. LCM might be combined with PB and VPA for patients with refractory epilepsy uncontrolled with monotherapy.

\section{REFERENCES}

1.Sperling MR, Klein P, Aboumatar S, et al. Cenobamate (YKP3089) as adjunctive treatment for uncontrolled focal seizures in a large, phase 3, multicenter, open-label safety study. Epilepsia 2020; 61: 1099-108. doi: 10.1111/epi.16525

2. Yamamoto T, Lim SC, Ninomiya $H$, et al. Efficacy and safety of perampanel monotherapy in patients with focal-onset seizures with newly diagnosed epilepsy or recurrence of epilepsy after a period of remission: The open-label Study 342 (FREEDOM Study). Epilepsia Open 2020; 5: 274-84. doi:10.1002/epi4.12398

3. Kobayashi K, Endoh F, Ohmori I, et al. Action of antiepileptic drugs on neurons. Brain Dev 2020; 42: 2-5. doi:10.1016/j.braindev.2019.07.006

4. Perucca E, Brodie MJ, Kwan P, et al. 30 years of second-generation antiseizure medications: impact and future perspectives. Lancet Neurol 2020; 19: 544-56. doi: 10.1016/S1474-4422(20)30035-1

5. Stephen LJ, Brodie MJ. Antiepileptic drug monotherapy versus polytherapy: pursuing seizure freedom and tolerability in adults. Curr Opin Neurol 2012; 25: 164-72. doi: 10.1097/WCO.0b013e328350ba68

6. Luszczki JJ, Czuczwar SJ. Biphasic characteristic of interactions between stiripentol and carbamazepine in the mouse maximal electroshockinduced seizure model: a three-dimensional isobolographic analysis. Naunyn Schmiedebergs Arch Pharmacol 2006; 374: 51-64. doi:10.1007/ s00210-006-0100-3

7.Luszczki JJ, Trojnar MK, Ratnaraj N, et al. Interactions of stiripentol with clobazam and valproate in the mouse maximal electroshockinduced seizure model. Epilepsy Res 2010; 90: 188-98. doi: 10.1016/j. eplepsyres.2010.04.006

8. Tallarida RJ. Quantitative methods for assessing drug synergism. Genes Cancer 2011; 2: 1003-8. doi: 10.1177/1947601912440575

9. Łuszczki JJ, Kondrat-Wróbel M, Zagaja M, et al. Sub-additive (antagonistic) interaction of lacosamide with lamotrigine and valproate in the maximal electroshock-induced seizure model in mice: an isobolographic analysis. Pharmacol Rep 2020. doi: 10.1007/s43440020-00117-y

10. Kondrat-Wróbel MW, Załuska K, Walczak A, et al. Antagonistic interaction of lacosamide with carbamazepine and valproate in the mouse tonic-clonic seizure model. Heal Probl Civiliz 2019; 13: 92-8. doi: 10.5114/hpc.2019.81105

11.Załuska K, Kondrat-Wróbel MW, Panasiuk-Poterek AN, et al. Synergy among oxcarbazepine, pregabalin and topiramate in the mouse maximal electroshock-induced seizure test - an isobolographic analysis. J PreClin Clin Res 2018; 12: 111-6. doi: 10.26444/jpccr/101578

12.Luszczki JJ, Zagaja M, Miziak B, et al. Beneficial combination of lacosamide with retigabine in experimental animals: an isobolographic analysis. Pharmacology 2018; 101: 22-8. doi: 10.1159/000480019 
13. Percie du Sert N, Hurst V, Ahluwalia A, et al. The ARRIVE guidelines 2.0: Updated guidelines for reporting animal research. Exp Physiol 2020. doi: 10.1113/EP088870

14. Stepien KM, Tomaszewski M, Luszczki JJ, et al. The interactions of atorvastatin and fluvastatin with carbamazepine, phenytoin and valproate in the mouse maximal electroshock seizure model. Eur J Pharmacol 2011; 674: 20-6. doi: 10.1016/j.ejphar.2011.10.030

15. Nieoczym D, Łuszczki JJ, Czuczwar SJ, et al. Effect of sildenafil on the anticonvulsant action of classical and second-generation antiepileptic drugs in maximal electroshock-induced seizures in mice. Epilepsia 2010; 51: 1552-9. doi: 10.1111/j.1528-1167.2009.02485.x

16. Litchfield JT, Wilcoxon F. A simplified method of evaluating dose-effect experiments. J Pharmacol Exp Ther. 1949; 96: 99-113.

17. Luszczki JJ, Filip D, Czuczwar SJ. Additive interactions of pregabalin with lamotrigine, oxcarbazepine and topiramate in the mouse maximal electroshock-induced seizure model: A type I isobolographic analysis for non-parallel dose-response relationship curves. Epilepsy Res. 2010; 91: 166-75. doi: 10.1016/j.eplepsyres.2010.07.009

18. Luszczki JJ, Andres MM, Czuczwar P, et al. Pharmacodynamic and pharmacokinetic characterization of interactions between levetiracetam and numerous antiepileptic drugs in the mouse maximal electroshock seizure model: An isobolographic analysis. Epilepsia. 2006; 47: 10-20. doi: $10.1111 / j .1528-1167.2006 .00364 . x$

19. Tallarida RJ. Drug combinations: tests and analysis with isoboles. Curr Protoc Pharmacol 2016; 72: 9.19.1-9.19.19. doi: 10.1002/0471141755. ph0919s72

20. Lederer S, Dijkstra TMH, Heskes T. Additive dose response models: explicit formulation and the Loewe additivity consistency condition. Front Pharmacol 2018; 9: 31. doi: 10.3389/fphar.2018.00031

21. Hitiris N, Mohanraj R, Norrie J, et al. Predictors of pharmacoresistant epilepsy. Epilepsy Res 2007; 75: 192-6. doi: 10.1016/j. eplepsyres.2007.06.003

22. Brodie MJ, Sills GJ. Combining antiepileptic drugs--rational polytherapy? Seizure 2011; 20:369-75. doi: 10.1016/j.seizure.2011.01.004

23. Perucca E. Pharmacological principles as a basis for polytherapy. Acta Neurol Scand Suppl. 1995; 162: 31-4. doi: 10.1111/j.1600-0404.1995. tb00497.x

24. Canevini MP, De Sarro G, Galimberti CA, et al. Relationship between adverse effects of antiepileptic drugs, number of coprescribed drugs, and drug load in a large cohort of consecutive patients with drugrefractory epilepsy. Epilepsia. 2010; 51: 797-804. doi: 10.1111/j.15281167.2010.02520.x
25. Palleria C, Di Paolo A, Giofrè C, et al. Pharmacokinetic drug-drug interaction and their implication in clinical management. J Res Med Sci 2013; 18: 601-10.

26. van Dijkman SC, Rauwé WM, Danhof M, et al. Pharmacokinetic interactions and dosing rationale for antiepileptic drugs in adults and children. Br J Clin Pharmacol 2018; 84: 97-111. doi: 10.1111/bcp.13400

27. Łuszczki JJ. Third-generation antiepileptic drugs: mechanisms of action, pharmacokinetics and interactions. Pharmacol Rep 2009; 61: 197-216. doi: 10.1016/s1734-1140(09)70024-6

28. Brodie MJ, Kwan P. Current position of phenobarbital in epilepsy and its future. Epilepsia. 2012; 53 Suppl 8: 40-6. doi:10.1111/epi.12027

29. Tomson T, Battino D, Perucca E. Valproic acid after five decades of use in epilepsy: time to reconsider the indications of a time-honoured drug. Lancet Neurol. 2016; 15: 210-8. doi: 10.1016/S1474-4422(15)00314-2

30. Kondrat-Wróbel MW, Łuszczki JJ. Interaction of three-drug combination of lacosamide, carbamazepine and phenobarbital in the mouse maximal electroshock-induced seizure model - an isobolographic analysis. Heal Probl Civiliz. 2016; 10: 55-61. doi: 10.5114/hpc.2016.58209

31. Kondrat-Wróbel MW, Łuszczki JJ. Additive interaction for three-drug combination of carbamazepine, lacosamide and lamotrigine against maximal electroshock-induced seizures - a type I isobolographic analysis. Eur J Clin Exp Med. 2017; 15: 303-9. doi: 10.15584/ ejcem.2017.4.1

32. Kondrat-Wróbel MW, Łuszczki JJ. Isobolographic additivity among lacosamide, lamotrigine and phenobarbital in a mouse tonic-clonic seizure model. Adv Clin Exp Med. 2018; 27: 881-6. doi: 10.17219/ acem/69132

33. Luszczki JJ. Isobolographic analysis of interaction for three-drug combination of carbamazepine, phenobarbital and topiramate in the mouse maximal electroshock-induced seizure model. Pharmacology 2016; 97: 259-64. doi: 10.1159/000444452

34. Luszczki JJ, Mazurkiewicz LP, Wroblewska-Luczka P, et al. Combination of phenobarbital with phenytoin and pregabalin produces synergy in the mouse tonic-clonic seizure model: An isobolographic analysis. Epilepsy Res. 2018; 145: 116-22. doi: 10.1016/j.eplepsyres.2018.06.003

35.Załuska K, Marzęda P, Bojar H, et al. Additive suppression of tonicclonic seizures in mice receiving the combination of carbamazepine, phenobarbital and valproate. J Pre-Clin Clin Res. 2019; 13: 72-5. doi: $10.26444 /$ jpccr/109381

36. Stephen LJ, Brodie MJ. Seizure freedom with more than one antiepileptic drug. Seizure 2002; 11: 349-51. doi: 10.1053/seiz.2002.0711

37. Stephen LJ, Forsyth M, Kelly K, et al. Antiepileptic drug combinations-have newer agents altered clinical outcomes? Epilepsy Res. 2012; 98: 194-8. doi: 10.1016/j.eplepsyres.2011.09.008 